\title{
ON A THEOREM OF FIGÀ-TALAMANCA
}

\author{
MARTIN E. WALTER ${ }^{1}$
}

\begin{abstract}
We give an example of a noncompact, unimodular group $G$ with the property that $B(G) \cap C_{0}(G)=A(G)$, where $A(G)$ is the Fourier algebra of $G, B(G)$ is the Fourier-Stieltjes algebra of $G$ and $C_{0}(G)$ is the set of all complex, continuous functions on $G$ vanishing at infinity. This example answers negatively a question raised by $\mathrm{A}$. Figà-Talamanca.
\end{abstract}

1. In a paper entitled Positive definite functions which vanish at infinity Alessandro Figà-Talamanca constructs a "singular" continuous, positive definite function, i.e., one not in the Fourier algebra of $G$, which vanishes at infinity for any locally compact unimodular group that satisfies the condition:

(H) The von Neumann algebra, $M(\lambda)$, generated by the left regular representation $\lambda$ of $G$ is not purely atomic.

Groups satisfying $(\mathrm{H})$ are of necessity not compact. In the aforementioned preprint, A.Figà-Talamanca notes that it is unknown whether or not unimodularity alone is sufficient (for a noncompact, locally compact group) to guarantee the existence of a "singular" continuous, positive definite function which vanishes at infinity. We show by means of an example in the following section that unimodularity alone is not sufficient to guarantee the existence of a positive definite function with the aforementioned properties.

2. In the fourth section of [3] the following group and its representation theory are discussed. We will briefly discuss this example with sufficiently many references so that the interested reader can fill in the details. Namely, let $G_{p}$ be the semidirect product, $N$ (s) $K$, where $N=\mathbf{Q}_{p}=p$-adic field (taken additively), $K=U=$ the compact (multiplicative) subgroup of $p$-adic units, and $K$ acts as a group of automorphisms of $N$ as follows: If $n \in N$, and $k \in K$, let $k(x)=k n$, the product in $\mathbf{Q}_{p}$. (See [9] for a complete discussion of $\mathbf{Q}_{p}, \mathbf{Z}_{p}, U$, etc.)

The "Mackey machine" works in this instance (cf. [8, pp. 42-43], [7], or [3]) to produce a complete description of $\hat{G}_{p}$, the continuous, irreducible, unitary representations of $G_{p}$. First there are the characters of $G_{p}$ which are trivial on $N$; this collection will be denoted by $\hat{K}$, as is done in [3]. Note that $\hat{K}$ corresponds to the orbit $\{0\}$ of $\hat{N}$ under the action induced by $K$. The other orbits, denoted by $N_{j}=\left\{n \in N\right.$ : "height" of $\left.n=p^{j}\right\}$, each give rise to an

Received by the editors December 29, 1975.

AMS (MOS) subject classifications (1970). Primary 43A50; Secondary 43A30, 43A35.

Key words and phrases. Fourier and Fourier-Stieltjes algebra, inverse Fourier transform for nonabelian groups, positive definite function, singular Fourier-Stieltjes series.

${ }^{1}$ This research was supported in part by NSF Grant GP-28697. 
irreducible, induced representation $T^{j}=U^{\chi_{j}}$ with $\chi_{j} \in N_{j}, j \in \mathbf{Z}$, the integers, where in the above we identify $N$ with its character group. Thus $\hat{G}_{p}=\hat{K} \cup \cup_{j \in \mathbf{Z}}\left\{T^{j}\right\}$, and the left regular representation of $G_{p}$,

$$
\lambda=\sum_{j=-\infty}^{+\infty} \oplus \infty T_{j},
$$

a direct sum where each $T_{j}$ is counted with infinite multiplicity. The topology of $\hat{G}_{p}$ is discussed in $[3, \S 4]$.

We thus have

Proposition 1. A continuous, unitary representation $\pi$ of $G_{p}$ is disjoint from $\lambda$ if and only if there exists a representation $\pi_{0}$ of $K$ such that $\pi=\pi_{0}^{\circ} q$, where $q(n, k)=k,(n, k) \in G_{p}$.

REMARKS. The symbol $\circ$ denotes composition. The proof of this proposition is similar to that of (4.34) of [1].

Proof. Since $G_{p}$ is separable we can without loss of generality assume that $H_{\pi}$, the Hilbert space of $\pi$, is separable. Suppose that $\pi$ is disjoint from $\lambda$. Since $\lambda=\sum_{j=-\infty}^{\infty} \oplus \infty T^{j}$, no $T^{j}$ is equivalent to a subrepresentation of $\pi$. Since $G_{p}$ is C.C.R. (cf. [3]), hence, in particular, $C^{*}\left(G_{p}\right)$ is postliminaire, we can apply $[4,8.6 .5,8.6 .6]$, and express $\pi$ as a direct integral, $\pi=\int_{G_{p}}^{\oplus} \pi_{\zeta} d \mu(\zeta)$, where $\mu$ is a positive measure on $\hat{G}_{p}$ provided with its usual Borel structure and $\pi_{\zeta}$ is a multiple of a representation of the class $\zeta$. Note that the direct integrals are really just direct sums since $\hat{G}_{p}$ is countable. Now by $[4,8.6 .8]$, a $T^{j}$ is not contained in the sum for $\pi$ if and only if $\mu\left(\left\{T^{j}\right\}\right)=0$, which means that the support of $\mu$ is contained in $\hat{K}$. Thus $\pi=\int_{K}^{\Phi} \pi_{k} d \mu(k)$, where $\pi_{k}$ is trivial on $N$, hence $\pi=\pi_{0} \circ q$ for a continuous, unitary representation $\pi_{0}$ of $K$.

Conversely, suppose there is a continuous, unitary representation $\pi_{0}$ of $K$ such that $\pi=\pi_{0} \circ q$, where again without loss of generality $H_{\pi}=H_{\pi_{0}}$ is separable. We can express $\pi_{0}$ as a direct integral (sum) $\pi_{0}=\int_{K}^{\Phi_{K}} \pi_{k} d \mu_{0}(k)$, and thus $\pi=\int_{G}^{\oplus} \pi_{\zeta} d \mu(\zeta)$, where $\mu$ is a positive measure with support $\mu$ contained in $\hat{K}$. (The values of $\pi_{\zeta}$ for $\zeta \notin \hat{K}$, of course, are immaterial.) Thus $\pi$ is disjoint from all $T^{j}$, hence from $\lambda$.

DEFINITION. Let $B_{s}\left(G_{p}\right)$ be the two-sided, translation invariant, Banach subspace of $B\left(G_{p}\right)$ consisting of all coefficients of all representations disjoint from $\lambda$. In the notation of [10], $B_{s}\left(G_{p}\right)=(z[\omega]-z[\lambda]) . B\left(G_{p}\right)$, where $z[\omega]$ is the support in $W^{*}\left(G_{p}\right)$ of $\omega$, the universal representation; and $z[\lambda]$ is the support in $W^{*}\left(G_{p}\right)$ of $\lambda$. Recall that $A\left(G_{p}\right)=z[\lambda]$. $B\left(G_{p}\right)$ (cf. [5]), and that $z[\omega]=e$, the identity in $W^{*}\left(G_{p}\right)$.

COROllary 1. (i) $B\left(G_{p}\right)=A\left(G_{p}\right) \oplus B_{s}\left(G_{p}\right)$.

(ii) $B_{s}\left(G_{p}\right)$ is isometrically isomorphic with $A(K)=B(K)$. In particular, $B_{s}\left(G_{p}\right)$ is a Banach algebra whose elements are constant on cosets of $N$.

(iii) $z[\omega]-z[\lambda] \in \sigma\left(B\left(G_{p}\right)\right)$, the spectrum of $R(G)$

Proof. Statement (i) is evident from the definition of $B_{s}\left(G_{p}\right)$. Statement (ii) follows from Proposition 1, since $b \in B_{s}\left(G_{p}\right)$ if and only if it is a coefficient of a representation that factors via $q$, see also [5, (2.20)]. Statement (iii) follows from [11, Proposition 1]. We observe in passing that $z[\omega]-z[\lambda]$ is 
critical in the sense of $\left[11\right.$, p. 275] and equals $z_{F}[11$, p. 276].

Corollary 2. $A\left(G_{p}\right)=B\left(G_{p}\right) \cap C_{0}\left(G_{p}\right)$.

Proof. If $b \in B\left(G_{p}\right)$, by Corollary $1, b=a+b_{s}$, with $a \in A\left(G_{p}\right), b_{s} \in$ $B_{s}\left(G_{p}\right)$. If $b$ vanishes at infinity, however,

$$
\lim _{n \rightarrow \infty}(b-a)(n, k)=0=\lim _{n \rightarrow \infty} b_{s}(n, k)=b_{s}(n, k),
$$

since $b_{s}$ is constant on cosets of $N$. Thus $b_{s}=0$, and $b \in A\left(G_{p}\right)$.

Now $G_{p}$ is unimodular by [2, Proposition 2.1-C], and $G_{p}$ does not satisfy condition $(\mathrm{H})$. We see from Corollary 2 that $G_{p}$ has no singular, continuous, positive definite functions which vanish at infinity. Thus unimodularity alone will not suffice to guarantee the conclusion of A. Figà-Talamanca's theorem in the absence of condition $(\mathrm{H})$.

We remark in closing that the present author has difficulty verifying a certain claim near the end of [6], even assuming condition $(H)$. This situation, of course, does not affect the example presented above.

NotE ADDED IN PROOF. Professor Figà-Talamanca informs us that he has corrected the errors in [6] and that Giancarlo Mauceri has obtained our result independently.

\section{REFERENCES}

1. G. Arsac, Sur l'espace de Banach engendré par les coefficients d'une representation unitaire, Thèse, L'Université Claude-Bernard-Lyon, 1973.

2. L. Baggett, $A$ description of the topology on the dual spaces of certain locally compact groups, Trans. Amer. Math. Soc. 132 (1968), 175-215.

3.,$A$ separable group having a discrete dual is compact, J. Functional Analysis 10 (1972), 131-148. MR 49 \#10816.

4. J. Dixmier, Les $C^{*}$-algèbres et leurs représentations, 2 ième ed., Gauthier-Villars, Paris, 1969. MR 39 \#7442.

5. P. Eymard, L'algèbre de Fourier d'un groupe localement compact, Bull. Soc. Math. France 92 (1964), 181-236. MR 37 \#208.

6. A. Figa-Talamanca, Positive definite functions which vanish at infinity (preprint).

7. G. Mackey, Unitary representations of group extensions. I, Acta Math. 99 (1958), 265-311. MR $20 \# 4789$.

8. - Induced representations of groups and quantum mechanics, Benjamin, New York, 1968.

9. J. Serre, Cours d'arithmétique, Presses Universitaires de France, Paris, 1970. MR 41 \# 138.

10. M. E. Walter, $W^{*}$-algebras and nonabelian harmonic analysis, J. Functional Analysis 11 (1972), 17-38. MR 50 \#5365.

11. , On the structure of the Fourier-Stieltjes algebra, Pacific J. Math. 58 (1975), 267-281.

Department of Mathematics, University of Colorado, Boulder, Colorado 80302 